PORTIONS OF THIS REPORT ARE IILEGIBLE. It

has been reproduced from thr seci a aible copy to permit the broadest possible avaitabllity.

CCNF- $8404156-3$

CONF-8 $200156--3$

). 05014050

\title{
A TIME-DEPENDENT MEAN-FIELD THEORY FOR PROMPT NUCLEON EMISSION \\ IN HEAVY-ION REACTIONS
}

\author{
A. S. Umar \\ Wright Nuclear Structure Laboratory, Yale University \\ New Haven, Connecticut 06511 \\ and \\ Oak Ridge National Laboratory \\ Oak Ridge, Tennessee 37831 \\ (International Conference on Fusion Reactions \\ Below the Coulomb Barrier) \\ Cambridge, Massachusetts \\ June 13-15, 1984
}

\begin{abstract}
By acceptance of this article, the publisher or recipient ackr.owledges the U.S. Government's right to retain a nonexclusive, royalty.free license in and to any copyright covering the article.
\end{abstract}

\section{DISCLAIMER}

This report was prepared as an account of work sponsored by an agency of the United States Government. Neither the United States Government nor any agency thereof, nor any of their employees, makes any warraniy, express or implied, or assumes any legal liability or responsibility for the accuracy, completeness, or urefulness of any information, apparatus, product, or process disclosed, or represents that its use would not infringe priyately owned rights. Reference herein to any specific commercial product, process, or service by trade name, trademark, manufacturer, or otherwise does not necessarily constitute or imply its endorsement, recommendation, or favoring by the United States Governmert or any agency thereof. The views and opinions of authors expressed herein do not necessarily state or reflect those of the United States Government or any agency thereor.

DISTARSUTON OF THIS DRSUMEET IS UMLIMITED 


\author{
A. S. Umar \\ Wright Nuclear Structure Laboratory, Yale University \\ New Haven, Connecticut 06511 \\ and \\ Oak Ridge National Laboratory \\ Oak Ridge, Tennessee 37831
}

In recent years the study of fast particles emitted during heavy-ion reactions has been one of the outstanding problems of nuclear physics. Here we present a meanfield theory for the problem. ' The essence of the model is to treat the relative motion of the ions classically while treating the internal excitations quantum mechanically. The motivation for the theory comes from the earlier time-dependent Hartree-Fock (TDHF) calculations. ${ }^{2}$ These calculations have shown that the time scaie for particle emission was on the order of $10^{-22}$ secs., and, in particular, these particles were emitted before dynamical rearrangement and exchange processes induce an appreciable change on the entrance channel values of the heavy-ion masses and energies. We consider particle emission from a reaction of the type $A+B \rightarrow N+X$, where $N$ denotes a singie emitted nucleon and $X$ stands for everything else. Here, for simplicity, we study particle emission from fragment $A$ which is perturbed by the external time-dependent field produced by a structureless particle of mass $B$ located at a distance $R$ from the center of $A$. The Hamiltonian which governs the system can be written as

$$
H\left(\left\{\vec{r}_{i}\right\}, \vec{R}\right)=H_{n}\left(\left\{\vec{r}_{i}\right\}\right)+T_{\vec{R}}+U\left(\vec{R},\left\{\vec{r}_{i}\right\}\right)
$$

where $H$ is the intrinsic Hartree-Fock Hamiltonian for $A$, depending on the intrinsic coordinates $\left\{\vec{r}_{i}\right\}$. $T$ is the relative kinetic energy of $A$ and $B$, and $U$ is an external one-body field which induces the coupling between the internal degrees of freedom of $A$ and the relative motion of $B$

$$
U\left(\vec{R},\left\{\vec{r}_{j}\right\}\right)=\sum u\left(\vec{r}_{i}-\vec{k}\right) \text {. }
$$

The one-body nature of $U$ is an assumption of our mode1. We approximate the wavefunction $\Psi$ associated with $H$ to be a product of two terms,

$$
\Psi\left(\left\{\vec{r}_{i}\right\}, \vec{R}, t\right)=G(\vec{R}, t) \Phi\left(\left\{\vec{r}_{i}\right\}, t\right)
$$

\footnotetext{
*The research was sponsored in part by the U.S. Department of Energy under contract DE-ACO2-ERÔ3074, and in part by the Division of Nuclear Physics, U.S. Department of Energy under contract DE-AC05-840R21400 with Martin Marietta Energy Systcms, InC.
} 
where $\Phi$ is a time-dependent Slater determinant of single-particle states $\phi_{\lambda}$ describing the motion of the particles in $A$, and $G$ is the wavefunction which gives the motion of $B$. Variation of the action with respect to $G^{*}$ and $\phi_{\lambda}^{\star}$ yields the dynamical equations for $\phi_{\lambda}$

$$
\left[h(\vec{r}, t)+\int d \vec{R}|G(\vec{R}, t)|^{2} u(\vec{r}-\vec{R})-i h \partial_{t}\right] \phi_{\lambda}(\vec{r}, t)=0
$$

and for $G$

$$
\left[T_{\vec{R}}+\int d \vec{r} \rho(\vec{r}, t) u(\vec{r}-\vec{R})-i h \partial_{t}\right] G(\vec{R}, t)=0 \text {. }
$$

Here $h$ and $\rho$ are the single-particle Hartree-Fock Hamiltonian and the single-particle density for $A$. The solution of $E q .(2)$ is obtained, in the classical limit, by using wavepackets which are localized on classical trajectories. He also calculate oneand two-particle inclusive, invariant cross sections as ${ }^{3}$

$$
\begin{aligned}
& \frac{d^{3} \sigma^{(1)}}{d k^{3}}=\frac{1}{(2 \pi)^{2}} \sum_{\lambda=1}^{A} \int_{0}^{b_{\max }} b d b\left|c_{\vec{k} \lambda}(\infty)\right|^{2} \\
& \frac{d^{6} \sigma^{(2)}}{d^{3} k d^{3} q}=\frac{1}{(2 \pi)^{5}} \int_{0}^{b} \max d b \quad b \sum_{\lambda, \mu=1}^{A}\left|c_{\lambda \vec{k}}\right|^{2}\left|c_{\mu \vec{q}}\right|^{2}-\left.\left|\sum_{\lambda=1}^{A} c_{\lambda \vec{k}}^{*} c_{\lambda \vec{q}}\right|\right|^{2} \mid .
\end{aligned}
$$

In the above expressions, $b$ denotes the impact parameter, and the $C$ variables are the expansion coefficients of $\phi_{\lambda}$ in terms of the bound and continuum states ${ }^{4}$ of $h_{0}$, which measure the occupation-probability transfer from bound to continuum states. For these calculations, we have used a phenomenological external field which was a rankone, separable potential. For the deep-inelastic branch of the $160+{ }^{93} \mathrm{Nb}$ reaction at $E_{1 a b}=204 \mathrm{MeV}$ the range was adjusted so as to contain the interaction within the strong absorption radius of this system, and the adjustment of the strength gave us a multiplicity of 0.134 in agreement with the corresponding experimental value. 5 The $s$-wave contribution from 160 to the following results is less than $1 \%$ in agreement with the previous TDHF result. We have used a Coulomb trajectory generated by a modified Coulomb potential. We calculate the mean velocitj of the frame from which the neutrons are emitted as

$$
V_{b}=\int_{-\infty}^{+\infty} d t p(t) V_{T}(t) \int_{-\infty}^{+\infty} d t p(t)
$$

where $V_{T}$ is the relative velocity of the ions on the trajectory, and $p(t)$ is the total emission probability in time. The average of $V_{b}$ over all trajectories yields a moving source velocity of $0.124 \mathrm{c}$. In Fig. 1 we show the contours of the invariant singles cross section as a function of longitudinal and transverse velocities. From this figure we can deduce the following observations: The contours are centered around the moving source velocity, and they have the characteristic exponential dependence on the total velocity. The energy-angle cross sections are shown in Fig. 2 as a function of energy and angle. The experimental results are also shown, and we see a fairly good agreement with the data except at $63^{\circ}$. This disagreement may be 


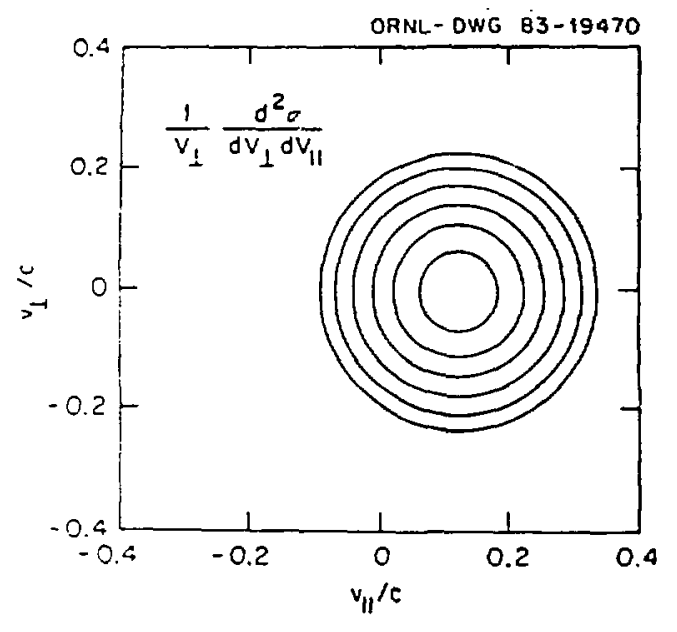

Fig. 1. Contours of the invariant cross section as a function of transverse and longitudinal velocities.

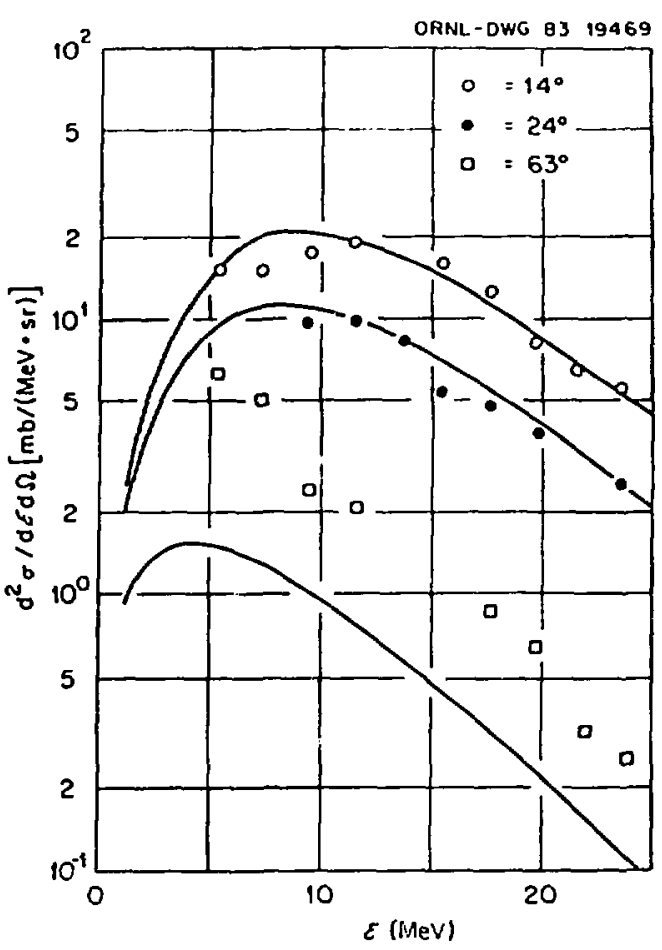

Fig. 2. Double differential cross sections as a function of laboratory energy for angles $14^{\circ}, 24^{\circ}$, and $63^{\circ}$.

due to the exclusion of the emission from $\mathrm{Nb}$ in our calculations. In order to extract a temperature parameter, we have fitted the angle integrated energy spectrum to the functional form

$$
\frac{d \sigma}{d \varepsilon} \cong N\left(\frac{\varepsilon}{T}\right)^{\alpha} e^{-\varepsilon / T}
$$

with $\mathrm{N}=69.3 \mathrm{fm}^{2} \mathrm{MeV}, T=5.2 \mathrm{MeV}$, and $\alpha=1.32$. In Fig. 3 we show the two-particle cross section of $\mathrm{Eq} .(3 \mathrm{~b})$ as a function of the relative two-neutron velocity. Since we have derived a mean-field theory, it is important to understand the source of these correlations. First, the wavefunction of the nucleus is at all times a slater determinant. The antisymmetry of the wavefunction leads to the second term on the righthand side of $\mathrm{Eq}$. $(3 \mathrm{~b})$. The second type of correlations are the dynamical correlations. There is yet another source of correlations which is not in our model. If two nucleons are emitted in nearly the same direction, then they will interact substantially before they reach the detector. Since this interaction will be dominated by the deuteron pole, we have used the separable interaction of Yamaguchi ${ }^{6}$ as our residual interaction. The dashed line of Fig. 3 shows the effect of this final-state interaction upon the two-neutron cross section. As we see, the effect of a residual interaction leaves the momentum localization unchanged. The naive application of the uncertainty relation gives us an equivalent space localization of $2.5 \mathrm{fm}$ which is approximately the size of 160 . These results show the similar characteristics of 
two-particle correlations obtained for other systems. ${ }^{7,8}$

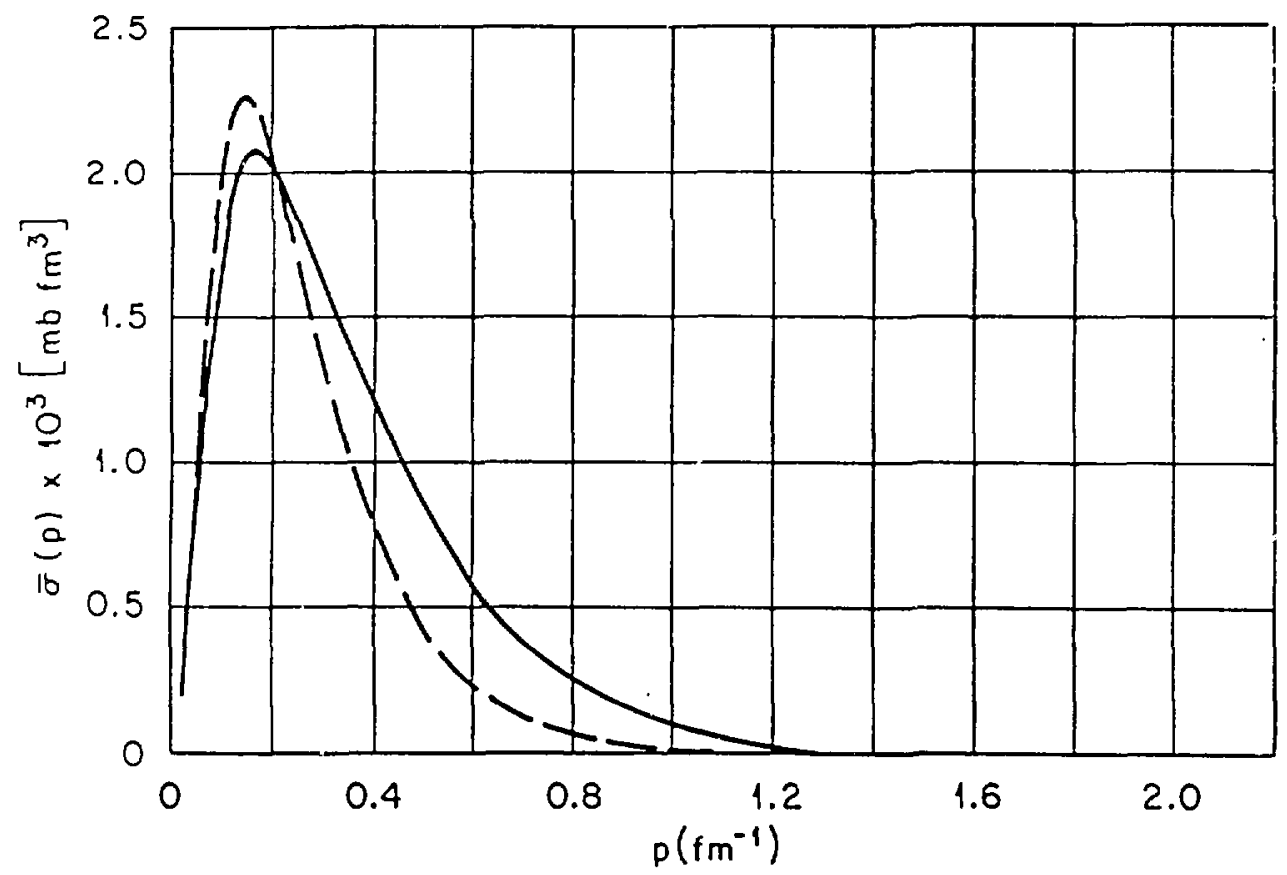

Fig. 3. Two-neutron cross section of Eq. (3b) as a function of relative neutron momentum (solid line) and the same curve with the addition of a final-state interaction (dashed curve). The dashed curve is divided by 100 .

References:

1. A. S. Umar, M. R. Strayer, and D. J. Ernst, Phys. Lett. B (in press).

2. K. R. S. Devi, M. R. Strayer, K. T. R. Davies, S. E. Koonin, and A. K. Dhar, Phys. Rev. C 24, 2521 (1981).

3. A. S. Umar, M. R. Strayer, D. J. Ernst, and K. R. S. Devi (to be published)。

4. W. H. Bassichis and M. R. Strayer. Phys. Rev. C 20, 915 (1979).

5. A. Gavron, R. L. Ferguson, F. E. Obenshain, F. Plasil, G. R. Young, D. G. Sarantites, and C. F. Maguire, Phys. Rev. Lett. $\underline{46}, 8$ (1981).

6. Y. Yamaguchi, Phys. Rev. $\underline{95}, 1628$ (1954).

7. S. E. Koonin, Phys. Lett. 70B, 43 (1977).

8. W. G. Lynch, C. B. Chitwood, M. B. Tsang, D. J. Fields, D. R. Klesch, C. K. Gelbke, G. R. Young, T. C. Awes, R. L. Ferguson, F. E. Obenshain, F. Plasil, R. L. Robinson, and A. D. Panagiotu, Phys. Rev. Lett. 51, 1850 (1983). 